\title{
Cutaneous Solution in Single-dose Container
}

National Cancer Institute

\section{Source}

National Cancer Institute. Cutaneous Solution in Single-dose Container. NCI Thesaurus.

Code C149416.

Medicinal product consisting of a cutaneous solution presented in a single-dose container. 